\title{
Introduction to the special issue of the ECML PKDD 2020 journal track
}

\author{
Ira Assent ${ }^{1}$ - Carlotta Domeniconi ${ }^{2} \cdot$ Aristides Gionis $^{3} \cdot$ Eyke Hüllermeier $^{4}$ \\ Published online: 8 September 2020 \\ (๑) The Author(s), under exclusive licence to Springer Science+Business Media LLC, part of Springer Nature 2020
}

The papers contained in this special issue have been accepted for the ECML PKDD 2020 journal track, which allows authors to combine a journal publication with a conference presentation of their work. The journal track was launched in 2013 and has accompanied the European Conference on Machine Learning and Knowledge Discovery in Databases (ECML PKDD) since then. It solicits high quality papers combining the timeliness and novelty of conference contributions with the maturity and sophistication of journal publications-survey papers or extensions of previously published conference papers are normally excluded. Authors can submit to the Machine Learning Journal or the Data Mining and Knowledge Discovery Journal. This year, the journal track offered four submission deadlines between September 2019 and May 2020. Accepted papers were presented (virtually) at the ECML PKDD 2020 conference in Ghent, Belgium, from September 14-18, 2020.

The Machine Learning journal received a total of 71 submissions, of which 9 were accepted in time for this special issue. The special issue also contains 2 papers, edited by Karsten Borgwardt, Po-Ling Loh, Evimaria Terzi, and Antti Ukkonen, which were submitted to the journal track for ECML PKDD 2019 but not accepted in time. The contributions to this issue cover a wide spectrum of topics in machine learning, ranging from deep learning and Bayesian optimization to model interpretability.

We thank all authors who submitted papers to the journal track, as well as the members of our Guest Editorial Board and other reviewers who provided timely and high-quality reviews. All papers went through a rigorous reviewing process meeting the standards of the Machine Learning Journal, and most of them have only been accepted after careful revision by the authors. We are especially grateful to Julie Rasmussen and Malene Andersen from Aarhus University, who coordinated the reviewing efforts. We will honor outstanding reviewing service to this special issue through Reviewer Awards, whose winners will be announced on the ECML PKDD 2020 website. We are also grateful for the support of Peter Flach (Editor-in-Chief of the Machine Learning journal), Johannes Fürnkranz (Editor-in-Chief of the Data Mining and Knowledge Discovery journal), and Melissa Fearon

Carlotta Domeniconi

cdomenic@gmu.edu

1 Aarhus University, Aarhus C, Denmark

2 George Mason University, Fairfax, USA

3 KTH Royal Institute of Technology, Stockholm, Sweden

4 Paderborn University, Paderborn, Germany 
(Executive Editor of Springer responsible for these journals). Finally, we thank Tijl De Bie, the ECML PKDD 2020 general chair, and the ECML PKDD 2019 journal track chairs for their guidance throughout the year. We hope that the readers enjoy the papers in this issue.

Publisher's Note Springer Nature remains neutral with regard to jurisdictional claims in published maps and institutional affiliations. 